\title{
Effect of AgI on Conduction Mechanism in Silver-Vanadate Superionic Glasses
}

\author{
Poonam Sharma, D. K. Kanchan*, Meenakshi Pant, Manish S. Jayswal, Nirali Gondaliya \\ Solid Sate Ionics \& Glass Research Laboratory, Department of Physics, Faculty of Science, The M.S. University of Baroda, Vado- \\ dara (Gujarat), India. \\ Email: *d_k_kanchan@yahoo.com
}

Received June $14^{\text {th }}, 2011$; revised July $27^{\text {th }}, 2011$; accepted August $9^{\text {th }}, 2011$.

\begin{abstract}
A quaternary super-ionic glass system $x \mathrm{AgI}$ : $(95-x)\left[\mathrm{Ag}_{2} \mathrm{O}: 2 \mathrm{~V}_{2} \mathrm{O}_{5}\right]: 5 \mathrm{TeO}_{2}$, where $40 \leq x \leq 65$ in steps of 5 , has been prepared by melt quenching technique. The prepared glass samples are characterized by X-ray, FTIR and DSC studies. As revealed by the FTIR spectra, the oxyanion network is not affected by the addition of AgI. The frequency dependence of the electrical conductivity for various glass compositions at different temperatures has been analyzed in terms of Jonscher's universal power law. The measurements reveal that the conductivity increases from $\sigma=7.62 \times 10^{-7} \mathrm{~S} / \mathrm{cm}$ to 1.15 $\times 10^{-4} \mathrm{~S} / \mathrm{cm}$ with increasing AgI content. The temperature dependent conductivity obeys the Arrhenius relationship. The impedance and modulus studies indicate the non-debye type of the frequency dispersion for all the glass samples.
\end{abstract}

Keywords: Conductivity, Glass Transition Temperature, Infrared Spectra, Impedance, Modulus

\section{Introduction}

$\mathrm{AgI}$ and Ag oxysalt based ion conducting materials attracted much attention from last many years, because of their high ionic conductivity at room temperature [1-4]. The glasses formed by AgI and $\mathrm{Ag}$ oxysalt complexes are constituted by randomly oriented micro domains made by arrays of tetrahedral oxysalt complexes [5]. These complexes are linked together and are surrounded by nonmobile $\mathrm{Ag}^{+}$ions which coordinate iodide polyhedra containing mobile $\mathrm{Ag}^{+}$ions. The conductivity maximum is realized when the oxyanions are coordinated with the largest number of iodide polyhedral which is compatible with the vitreous state. In general, it is found that the ionic conductivity increases with the AgI content in the glass composition. Although AgI based glasses have been studied widely, very limited studies have been reported on silver-based vanado-tellurite glasses [6]. Hence, we have used $\mathrm{V}_{2} \mathrm{O}_{5}$ and $\mathrm{TeO}_{2}$ as two glass formers with a very low amount of $\mathrm{TeO}_{2}$ salt (only $5 \mathrm{wt} \%$ ) as it is difficult to obtain glasses with high $\mathrm{V}_{2} \mathrm{O}_{5}$ contents and small amount of $\mathrm{TeO}_{2}$ is reported to induce superionic behavior when it is doped with metal halide [7]. Montani et. al. [8] had shown that addition of the $\mathrm{Ag}_{2} \mathrm{O}$ (network modifier) to the electronic $\mathrm{V}_{2} \mathrm{O}_{5}$ and $\mathrm{TeO}_{2}$ glass results the blocking of the electronic paths which causes the electronic conductivity to fall down. And increasing network modifier concentration gives rise to more ionic transport due to closeness of non-bridging oxygens.

The objective of present work is to investigate the influence of $\mathrm{AgI}$ salt content on conduction mechanism and ionic relaxation behavior in $\mathrm{Ag}_{2} \mathrm{O}-\mathrm{V}_{2} \mathrm{O}_{5}-\mathrm{TeO}_{2}$ glass system in framework of the modulus formalism i.e., the conductivity relaxation mechanism. In order to view this effect, we have prepared $\mathrm{xAgI}-(95-\mathrm{x})\left[\mathrm{Ag}_{2} \mathrm{O}: 2 \mathrm{~V}_{2} \mathrm{O}_{5}\right]$ $5 \mathrm{TeO}_{2}$ glass system, where $40 \leq \mathrm{x} \leq 65$ in steps of 5 in the present paper.

\section{Experimental}

Analytical Reagent grade starting chemicals: $\mathrm{AgI}, \mathrm{Ag}_{2} \mathrm{O}$, $\mathrm{V}_{2} \mathrm{O}_{5}$ and $\mathrm{TeO}_{2}$ were used to prepare the samples. All the compositions were weighed according to their mol\%, crushed and then ground in an agate mortar and pestle for 2 hours by wet grinding method. The homogenous mixture obtained was then kept in an alumina crucible in a controlled electric muffle furnace at $673 \mathrm{~K}$. Subsequently, the furnace was heated to $673 \mathrm{~K}$ at a rate of $100 \mathrm{~K} / \mathrm{h}$ and the melt was kept for $4 \mathrm{~h}$ at that temperature. After 4 hours, the melt was poured on a heavy thick copper plate kept at room temperature and pressed by another similar copper plate to quench it.

$\mathrm{X}$-ray diffraction was carried by X-ray diffraction analyzer (Shimadzu) at $2^{0} / \mathrm{min}$ scan rate to confirm the amorphous nature of prepared glass samples. The glass transition temperature of the amorphous samples was measured 
by Differential Scanning Calorimeter (DSC), TA Instruments (Model MBSE-2910) at a heating rate of $10 \mathrm{~K} / \mathrm{min}$. Fourier Transform Infrared (FTIR) spectra were measured in the range of $400-1100 \mathrm{~cm}^{-1}$ by a conventional $\mathrm{KBr}$ pellet method using an FTIR spectrophotometer (Bruker Model Vertex 70).

Electrical properties were measured by using an Impedance / Gain Phase analyzer (Solartron 1260) in the 10 $\mathrm{MHz}$ to $10 \mathrm{~Hz}$ frequency range at temperatures from room temperature to the glass transition temperature. The measurements were made by two-probe method in which quenched glass samples of about $1 \mathrm{~mm}$ thickness and rectangular in shape were coated with silver paint to serve as electrodes. The samples were kept in contact with two polished, cleaned and spring-loaded copper electrodes.

\section{Result and Discussion}

\subsection{IR Spectra}

The room temperature IR spectra in the region 400 $1100 \mathrm{~cm}^{-1}$ for $\mathrm{x}=45,50$ and $60 \mathrm{~mol} \%$ are shown in Figure 1. The band observed at $497 \mathrm{~cm}^{-1}$ is assigned to $v_{\text {sym }}$ stretching vibrations of $\mathrm{V}-\mathrm{O}-\mathrm{V}$ bridge of $\mathrm{V}_{2} \mathrm{O}_{7}^{-4}$ group. The broad band in the range of $650-740 \mathrm{~cm}^{-1}$ may be due to the stretching vibrations of the $\mathrm{Te}-\mathrm{O}_{\mathrm{ax}}$ (axial), Te- $\mathrm{O}_{\mathrm{eq}}$ (equatorial) bonds in deformed $\mathrm{TeO}_{4}$ groups, stretching vibrations of $\mathrm{TeO}_{3}$ of $\mathrm{Te}_{2} \mathrm{O}_{5}$ units and symmetric stretching vibrations of $\mathrm{V}-\mathrm{O}-\mathrm{V}$ bonds. The band at $966 \mathrm{~cm}^{-1}$ is assigned to symmetric stretching vibrations of the $\mathrm{VO}_{2}$ groups of the $\mathrm{VO}_{4}$ polyhedra. A small kink observed at $1020 \mathrm{~cm}^{-1}$ corresponds to the vibrations of the non-bridging $\mathrm{V}=\mathrm{O}$ of the $\mathrm{VO}_{5}$ groups. We have observed no change in the structure with increasing the AgI concentration. Hence, from the IR spectra we have concluded that the network structure of prepared glass samples is formed of vanadate and tellurite oxides and it remains

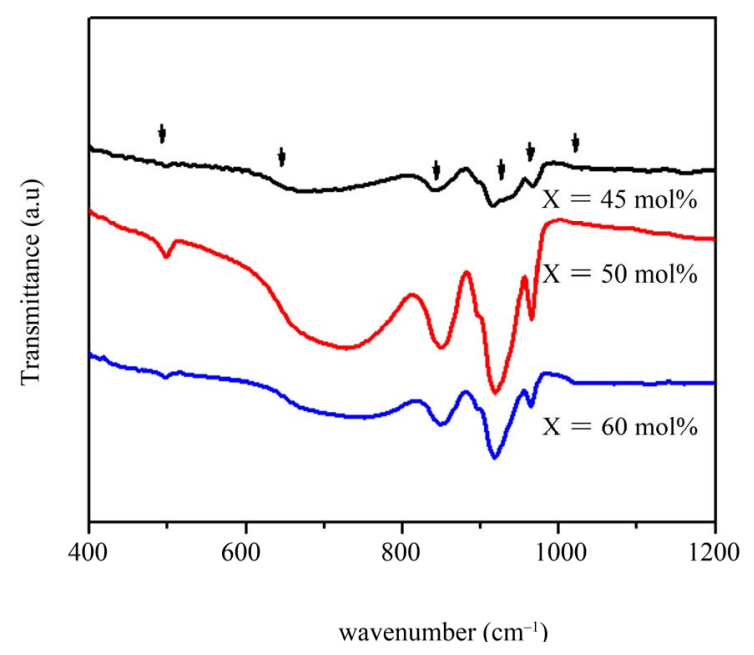

Figure 1. IR spectra for $x=45,50$ and $60 \mathrm{~mol} \%$. unaltered with AgI concentration. Similar results have been observed by several workers $[4,6]$.

\subsection{Impedance Plot}

Complex impedance data $Z^{*}$ is represented by its real, $Z^{\prime}$ and imaginary, $Z^{\prime \prime}$ components through the relation:

$$
Z^{*}=Z^{\prime}+i Z^{\prime \prime}
$$

Figure 2 shows the complex impedance plot for all glass compositions at $313 \mathrm{~K}$ and inset shows that of $\mathrm{x}=$ $45 \mathrm{~mol} \%$ at various temperatures. Figure shows the depressed semicircles which represent the presence of the distribution of relaxation times within the bulk response [9]. The high frequency semicircles are due to parallel combination of bulk resistance $\mathrm{R}_{\mathrm{b}}$ and bulk capacitance $C_{b}$ [10]. It is clear from the Figure 2 and its inset that by increasing the AgI concentration and temperature, the radius of the depressed semicircle decreases i.e., sample resistance decreases which may be due to the enhancement of the number of carrier ions and its mobility with temperature. Semicircle fits are used to determine the zero frequency impedance i.e., resistance and by using the known geometrical dimensions of the glass sample, the dc conductivity was determined.

\subsection{Conductivity}

The logarithmic dc conductivity $\sigma_{d c}$ of various glass compositions at $303 \mathrm{~K}$ is shown in Figure 3. It can be observed from figure that dc conductivity is increasing with AgI content. It is also observed that for all glass compositions the dc conductivity shows an activated behavior i.e., it increases with temperature. This behavior is characteristic of a thermally stimulated process and is attributed to increase of charge carrier energy with rise of temperature. It makes the hopping motion of charge carriers easier through the free energy barriers in the glass matrix. In addition to it, conductivity is also found to increase by three orders of magnitude with AgI concentration varying from $40 \mathrm{~mol} \%$ to $65 \mathrm{~mol} \%$. When a plot is made between dc conductivity versus activation energy for conduction, a linear relationship is observed (inset of Figure 3); the near linearity between the conductivity and the activation energy suggests the conductivity enhancement is directly related to the increasing the mobility of the charge carriers. As it appears, due to the increasing AgI concentration i.e. more $\mathrm{Ag}^{+}$ions, easy paths for the movement of the charge carriers are created and hence an increase in the conductivity is resulted with increase in AgI concentration in the prepared glass matrix [7].

The frequency dependent conductivity of the present glass samples has been measured in the frequency range from $10 \mathrm{MHz}$ to $10 \mathrm{~Hz}$ with the temperature range from $296 \mathrm{~K}$ to $353 \mathrm{~K}$. The conductivity is determined from the 
data of complex impedance values and calculated by using the relation

$$
\sigma=(t / a)\left(Z^{\prime} /\left(Z^{\prime 2}+Z^{\prime 2}\right)\right)
$$

The logarithmic conductivity as a function of frequency with different compositions at $323 \mathrm{~K}$ is shown in Figure 4 . The ac conductivity $\sigma_{a c}(\omega)$ for all the samples exhibit the same shape of the curve, with different conductivity values depending on the glass composition. The curve corresponds to bulk relaxation phenomenon, whereas the plateau region is connected with the dc conductivity $\left(\sigma_{d c}\right)$ of the glasses. The low frequency dispersion describes electrode-electrolyte interfacial phenomenon or space charge polarization [11].

As the frequency decreases, more and more charge accumulation occurs at the electrode and the electrolyte interface, which leads to a decrease in the number of mobile ions and eventually to a drop in conductivity at low frequency. In high frequency region, the mobility of charge carriers, $\mathrm{Ag}^{+}$, is high (near to relaxation time) and hence, the conductivity increases with frequency. Similar behavior is reported for other silver based ionic conductors [10-13].

At higher frequencies, the ac conductivity has been found to increase with frequency obeying Jonscher's Power Law [14] described by

$$
\sigma(\omega)=\sigma_{d c}+A \omega^{s}
$$

where $\sigma_{d c}$ is the dc conductivity of the sample, $A$ is a constant for a particular temperature, $\omega(=2 \pi f)$ is the angular frequency of the applied field and ' $s$ ' is the power law exponent in the range $0<s<1$. The power law exponent $s$ is a measure of degree of interaction with the environment. The value of $s>0$ is due to energy stored in the short range collective motion of ions and its higher value implies that large energy is stored in such collective motions. In present work, $s$ is found to increase with AgI content. The magnitude of $s$ appears to be

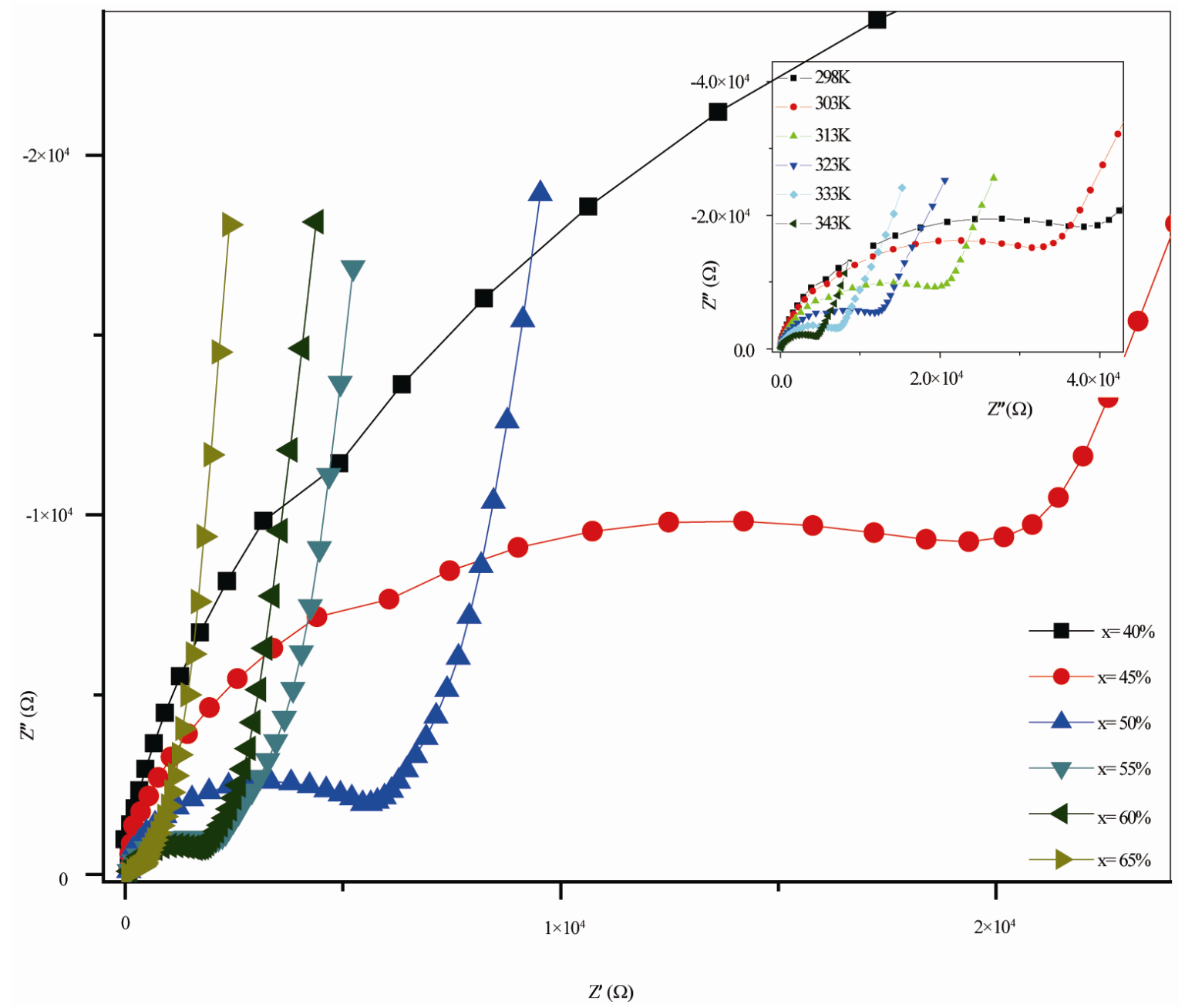

Figure 2. Impedance plot for different compositions at 313K and inset shows impedance plot at different temperatures for $\mathrm{x}=$ $45 \mathrm{~mol} \%$. 


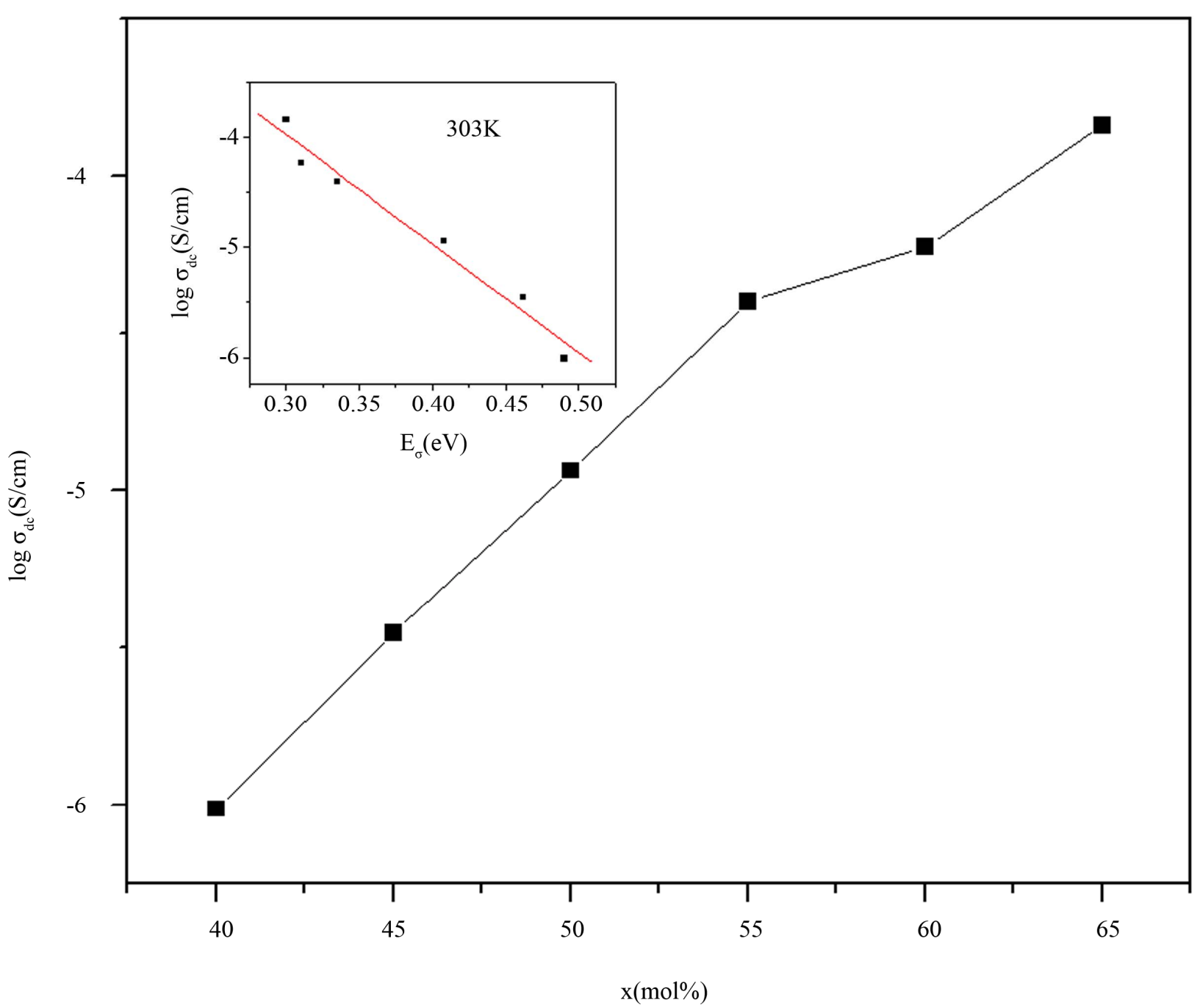

Figure 3. DC conductivity of AgI- $\mathrm{Ag}_{2} \mathrm{O}-\mathrm{V}_{2} \mathrm{O}_{5}-\mathrm{TeO}_{2}$ glass series and inset shows plot of activation energy vs. dc conductivity at $303 K$.

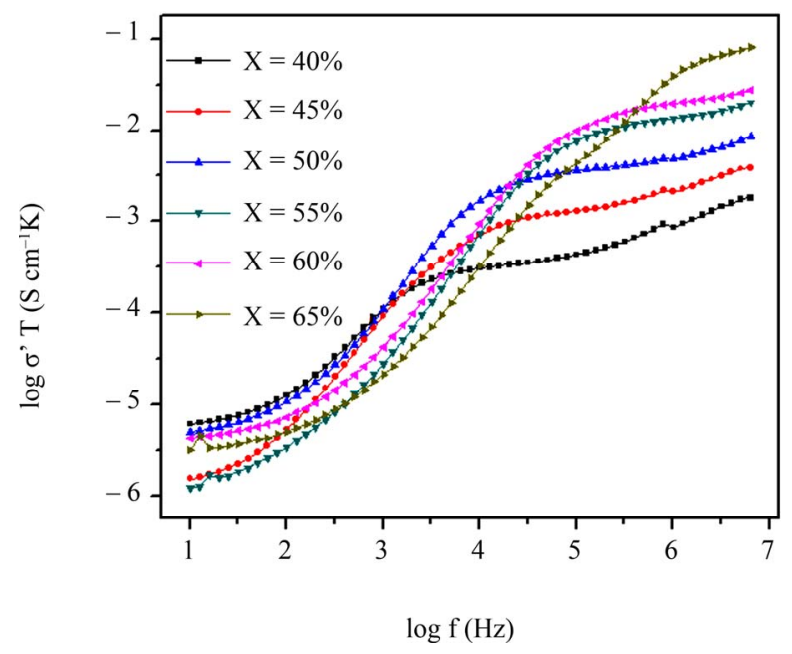

Figure 4. Frequency dependent conductivity for different compositions at $303 \mathrm{~K}$. associated with high degree of modifications. The hopping frequency $\omega_{p}$ is calculated by using Almond and West formula [15], frequency at which $\sigma^{\prime}(\omega)=2 \sigma_{d c}$. Since $\mathrm{Ag}^{+}$ions are the mobile species in the present case, $\omega_{p}$ has been identified as the hopping rate of $\mathrm{Ag}^{+}$ions. Usually $\omega_{p}$ is a characteristic frequency associated with the frequency of the dielectric loss peak and it is assumed to be thermally activated. It increases with the increases of temperature. It is, therefore, clear that the hopping rate of silver ions in the present system is also a thermally activated parameter. The mobile ion concentration factor $K^{\prime}$ has been evaluated as

$$
K^{\prime}=\sigma_{d c} T / \omega_{p}
$$

where $T$ is the temperature in Kelvin. The mobile ion concentration factor $K^{\prime}$ is found to be independent of temperature and is found to increase with AgI concentration. This shows that the increase in the mobile ion 
concentration is attributed to the increase in the conductivity i.e; with the increase in the AgI content.

\subsection{Modulus Formalism}

Modulus spectroscopy highlights the bulk effects and it is complementary to impedance spectroscopy which highlights electrode and grain-boundary effects. This formalism is particularly suitable to detect phenomena as electrode polarization and bulk property such as average relaxation time $[16,17]$. We have used the complex electric modulus formalism to analyze the relaxation processes in the present system as it discriminates against electrode polarization and other interfacial effects. Electric modulus can be represented by the following equation.

$$
M^{*}=j C_{0} Z^{*}
$$

In an ideal solid electrolyte system, it can be represented by a single parallel RC element, where $\mathrm{R}$ and $\mathrm{C}$ represent the resistance and capacitance, respectively and is characterized by a single time constant as conductivity relaxation time. In ideal system, the peak maximum of $Z^{\prime \prime}$ and $M^{\prime \prime}$ is found at the same frequency and the shape of the peaks are identical with that predicted by Debye theory [18]. With the appropriate scaling, the normalizedmodulus and impedance spectra of the Debye curves are completely superposable and are given by the equation:

$$
Z^{\prime \prime}=R \frac{\omega R C}{1+(\omega R C)^{2}} \quad ; \quad M^{\prime \prime}=\frac{C_{0}}{C} \times \frac{\omega R C}{1+(\omega R C)^{2}}
$$

The term, $\omega R C /\left[1+(\omega R C)^{2}\right]$ in imaginary part of impedance, $Z^{\prime \prime}$ and imaginary part of modulus, $M^{\prime \prime}$ is responsible for debye-like peak shapes. To understand the non-debye behavior of the prepared glass samples, $Z^{\prime \prime}$ and $M^{\prime \prime}$ at $303 \mathrm{~K}$ have been plotted in Figure 5 for $\mathrm{x}=60$ mol\% glass sample. It is observed from figure that the $Z^{\prime \prime} \max$ and $M^{\prime \prime}$ max do not occur at the same frequency which indicate a wide distribution of relaxation times. At low frequencies, $Z$ " shows large rise due to electrode

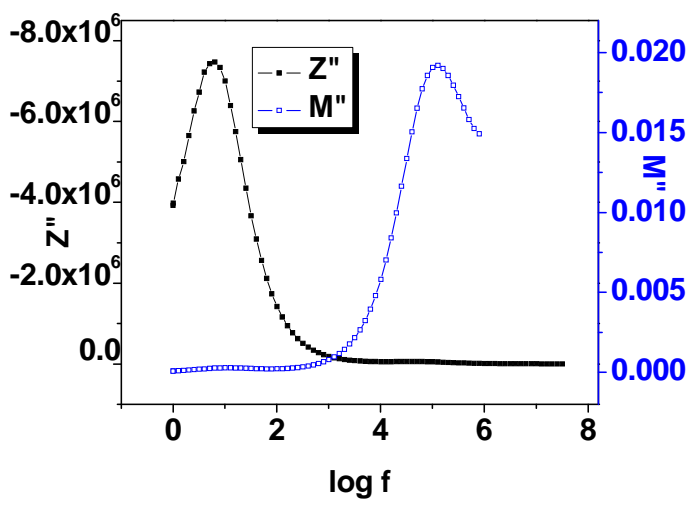

Figure 5. The impedance and modulus spectrum for $\mathbf{x}=$ $40 \mathrm{~mol} \%$ at $303 \mathrm{~K}$. polarization and at high frequency broadened modulus spectra indicates the distribution of relaxation times [19].

The real and imaginary part of modulus spectra for $\mathrm{x}=$ $45 \mathrm{~mol} \%$ at different temperatures is presented in Figure 6 (a) and Figure 6 (b) respectively. Other glasses also showed almost similar $M^{\prime}$ and $M^{\prime \prime}$ temperature dependence behavior. From $M^{\prime}$ graph, it shows that whatever be the temperature, the value of $M^{\prime}(\omega)$ reaches a constant value at higher frequencies. At low frequencies, $M^{\prime}$ and $M^{\prime \prime}$ approaches to zero indicating that the electrode polarization phenomenon make a negligible contribution to $M^{*}$ and may be ignored when the electric data has been analyzed in this form [20]. The observed long tail at low frequencies is due to the large capacitance associated with the electrodes.

The $M^{\prime \prime}(\omega)$ spectrum relative to a given temperature shows an asymmetrical peak approximately centered in the dispersion region of $M^{\prime}(\omega)$. The asymmetrical peaks obtained suggest that the material can be interpreted by an equivalent circuit composed of a single parallel RC element [18]. The left part of the peak corresponds to

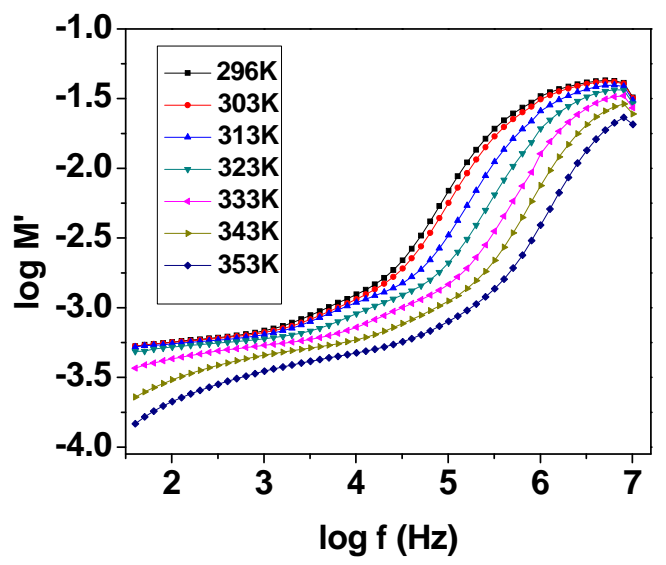

(a)

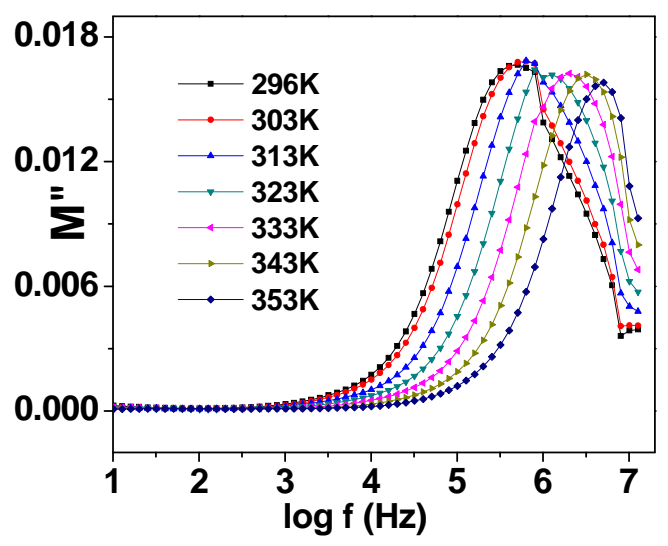

(b)

Figure 6. Frequency dependence of (a) real part, $M^{\prime}$ and (b) imaginary part of modulus, $M^{\prime \prime}$ at different temperatures for $x=45 \mathrm{~mol} \%$. 
long range mobility and the right part of the peak is attributed to ions spatially confined in narrow potential wells. The frequency range where the peak occurs indicates the transition between long and short range mobility and is defined by the condition $\omega_{c} \tau_{c}=1$ [21] where $\tau_{c}$ is the most probable relaxation time of ions. The $M^{\prime \prime}(\omega)$ peak height is found to be nearly same at different temperatures and it is observed to shift to a higher frequency with increasing temperature. The peak in $M^{\prime \prime}$ variation corresponds to the relaxation frequency and the corresponding relaxation time is systematically shifted to higher frequencies as the temperature increases.

Figure 7 shows the normalized plot of $M^{\prime \prime} / M^{\prime \prime}{ }_{\max }$ versus $\log f / f_{\max }$ of the modulus for the glassy system for $\mathrm{x}=$ $50 \mathrm{~mol} \%$ at different temperatures. The approximate overlap of the modulus curves for all temperatures indicates the dynamical processes occurring at different frequentcies are independent of temperature. Such results were also observed for other glass compositions. It is found that the normalized plot for different compositions do not merge on a single master curve which implies that the conductivity relaxation depends on the glass composition. This has usually been regarded as an indication of a distribution of relaxation times in the conduction process [20].The observed broad peak can be assigned to the summation of relaxations occurring in the bulk materials. The full width at half height obtained is greater than the Debye type of relaxation with single time constant is attributed to the presence of strong ion-ion interaction. The observed normalized plot is non-symmetric in agreement with the non-exponential behavior of the electrical function described by Kohlrausch-William-Watts (KWW) exponential function [22]

$$
\varphi(t)=\exp (-t / \tau)^{\beta} ;(0<\beta<1)
$$

where $\tau$ and $\beta$ are the conductivity relaxation time and Kohlrausch exponent respectively. The value of the Kohlrausch parameter $\beta$ for most practical solid electrolyte is

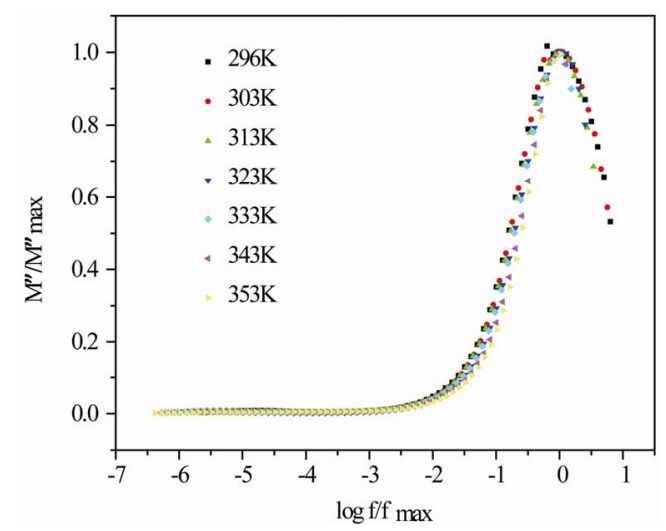

Figure 7. Normalized plot of $M^{\prime \prime}$ at different temperatures for $x=50 \mathrm{~mol} \%$. smaller than one. The calculated values of $\beta$ lies in the range of $0.6-0.9$ that is in agreement with the values reported [23].

\section{Conclusions}

The ionic conduction of the prepared glasses is a conesquence of the presence of glass modifier $\left(\mathrm{Ag}_{2} \mathrm{O}\right)$ and the dopant (AgI). IR spectra show that the network structure of prepared glass samples is formed of vanadate and tellurite oxides and remains unaltered with AgI concentration. The conductivity is found to obey the universal power law. The ionic conductivity as well as power law exponent is found to increase with AgI concentration. The modulus plot shows non-debye behavior and is asymmetric with respect to the peak maxima. The peaks are considerably broader on both sides of the maxima. The broadened modulus spectrum indicates the distribution of relaxation times in the conduction process. It is inferred from the normalized plot of the prepared glass samples that the conductivity relaxation is independent of temperature but composition dependent.

\section{REFERENCES}

[1] D. Dutta and A. Ghosh, "Dynamics of $\mathrm{Ag}^{+}$Ions in Binary Tellurite Glasses," Physical Review E, Vol. 52, No. 2, 2005, pp. 024201-024203.

doi:10.1103/PhysRevB.72.024201

[2] K. P. Padmasree and D. K. Kanchan, "Conductivity and Dielectric Studies on $20 \mathrm{CdI}_{2}-80\left[\mathrm{xAg}_{2} \mathrm{O}-\mathrm{y}\left(0.7 \mathrm{~V}_{2} \mathrm{O}_{5^{-}}\right.\right.$ $0.3 \mathrm{~B}_{2} \mathrm{O}_{3}$ ) Super Ion Conducting System Where $1 \leq \mathrm{x} / \mathrm{y} \leq 3$," Journal of Non-Crystalline Solids, Vol. 352, No. 36-37, 2006, pp. 3841-3848. doi:10.1016/j.jnoncrysol.2006.06.012

[3] J. E. Garbarczyk, P. Machowski, M. Wasiucionek and W. Jakubowski, "Electrical Properties of AgI- $\mathrm{Ag}_{2} \mathrm{O}-\mathrm{V}_{2} \mathrm{O}_{5}-\mathrm{P}_{2} \mathrm{O}_{5}$ Glasses," Solid State Ionics, Vol. 157, No. 1-4, 2003, pp. 269-273. doi:10.1016/S0167-2738(02)00220-5

[4] A. K. Arof and S. Radhakrishna, "Electrical Properties of Silver Vanadate Electrochemical Cells," Journal of Alloys and Compounds, Vol. 200, No. 1-2, 1993, pp. 129-134. doi:10.1016/0925-8388(93)90482-3

[5] T. Minami, "Preparation and Properties of Superionic Conducting Glasses Based on Silver Halides," Journal of Non-Crystalline Solids, Vol. 56, No. 1-3, 1983, pp. 15-26. doi:10.1016/0022-3093(83)90440-4

[6] M. Pant, D. K. Kanchan, P. Sharma and M. S. Jayswal, "Mixed Conductivity Studies in Silver Oxide Based Barium Vanado-Tellurite Glasses," Material Science and Engineering B, Vol. 149, No. 1, 2008, pp. 18-25. doi:10.1016/j.mseb.2007.11.037

[7] G. El-Damrawi, "Silver Ion Transporting Mechanism in Superionic Conducting AgI- $\mathrm{Ag}_{2} \mathrm{O}-\mathrm{TeO}_{2}$," Journal of Physics: Condensed Matter, Vol. 11, No. 33, 1999, pp. 6385-6394. doi:10.1088/0953-8984/11/33/308

[8] R. A. Montani, A. Lorente and A. M. Frechero, "Effect of 
$\mathrm{Ag}_{2} \mathrm{O}$ on the Conductive Behaviour of Silver Vanadium Tellurite Glasses," Solid State Ionics, Vol. 146, No. 2-3, 2002, pp. 323-327. doi:10.1016/S0167-2738(01)01023-2

[9] J. R. Macdonald, "Impedance Spectroscopy and its Use in Analyzing the Steady State Ac Response of Solid and Liquid Electrolytes," Journal of Electroanalytical Chemistry and Interfacial Electrochemistry, Vol. 223, No. 1-2, 1987, pp. 25-50. doi:10.1016/0022-0728(87)85249-X

[10] M. Pant, D. K. Kanchan and N. Gondaliya, "Transport Properties and Relaxation Studies in $\mathrm{BaO}$ Substituted $\mathrm{Ag}_{2} \mathrm{O}-\mathrm{V}_{2} \mathrm{O}_{5}-\mathrm{TeO}_{2}$ Glass System," Material Chemistry and Physics, Vol. 115, No. 1, 2009, pp. 98-104. doi:10.1016/j.matchemphys.2008.11.047

[11] M. D. Ingram, "Ionic Conductivity in Glass," Physics and Chemistry of Glasses, Vol. 28, 1987, pp. 215-234.

[12] J. Kawamura, S. Rikito, M. Shinya and M. Shimoji, “AC Conductivity of $\mathrm{Li}_{2} \mathrm{O}---\mathrm{Na}_{2} \mathrm{O}---\mathrm{B}_{2} \mathrm{O}_{3}$ Mixed-Alkali Glasses: Analysis Due to Transition Rate Distribution," Solid State Ionics, Vol. 25, No. 2-3, 1987, pp. 155-164. doi:10.1016/0167-2738(87)90115-9

[13] N. Satyanaryan, A. Karthikeyan and M. Venkateswarlu, "AC Conductivity Studies on the Silver Molybdo-Arsenate Glassy System," Journal of Material Science, Vol. 31, No. 20, 1996, pp. 5471-5477. doi:10.1007/BF01159319

[14] A. K. Jonscher, "The 'Universal' Dielectric Response," Nature, Vol. 267, 1977, pp. 673-679. doi:10.1038/267673a0

[15] D. P. Almond, C. K. Duncan and A. R. West, "The Determination of Hopping Rates and Carrier Concentrations in Ionic Conductors by a New Analysis of AC Conductivity," Solid State Ionics, Vol. 8, No. 2, 1983, pp. 159-164. doi:10.1016/0167-2738(83)90079-6

[16] D. P. Almond and A. R. West, "Impedance and Modulus Spectroscopy of Real Dispersive Conductors," Solid State
Ionics, Vol. 11, No. 1, 1983, pp. 57-64. doi:10.1016/0167-2738(83)90063-2

[17] J. M. Reau, X. Y. Jun, J. Senegas, C. L. Deit and M. Poulain, "Influence of Network Modifiers on Conductivity and Relaxation Parameters in Some Series of Fluoride Glasses Containing LiF," Solid State Ionics, Vol. 95, No. 3-4, 1997, pp. 191-197. doi:10.1016/S0167-2738(96)00564-4

[18] R. J. Grant, M. D. Ingram and A. R.West, "An Investigation of $\beta$-alumina Electrolytes by Electric Modulus Spectroscopy," Electrochimica Acta, Vol. 22, No. 7, 1977, pp. 729-734. doi:10.1016/0013-4686(77)80027-3

[19] J. M. Reau, S. Rossignol, B. Tanguy, J. M. Rojo, P. Herrero, R. M. Rojas and J. Sanz, "Conductivity Relaxation Parameters of Some $\mathrm{Ag}^{+}$Conducting Tellurite Glasses Containing AgI or the $(\mathrm{AgI})_{0.75}(\mathrm{~T} 1 \mathrm{I})_{0.25}$ Eutectic Mixture," Solid State Ionics, Vol. 74, No. 1-2, 1994, pp. 65-73. doi:10.1016/0167-2738(94)90438-3

[20] M. Sural, A. Ghosh, "Electric Conductivity and Relaxation in $\mathrm{ZnF}_{2}-\mathrm{AlF}_{3}-\mathrm{PbF}_{2}-\mathrm{LiF}$ Glasses," Solid State Ionics, Vol. 130, No. 3-4, 2000, pp. 259-266. doi:10.1016/S0167-2738(00)00641-X

[21] P. B. Macedo, C. T. Moynihan and R. Bose, "The Role of Ionic Diffusion in Polarization in Vitreous Ionic Conductors," Physics and Chemistry of Glasses, Vol. 13, 1972, pp. 171-179.

[22] G. Williams and D. C. Watts, "Non-Symmetrical Dielectric Relaxation Behavior Arising from a Simple Empirical Decay Function," Transactions of the Faraday Society, Vol. 66, 1970, 80-85, doi:10.1039/TF9706600080.

[23] A. Pan and A. Ghosh, "Dynamics of Lithium Ions in Bismuthate Glasses," Journal of Chemical Physics, Vol. 112, No. 3, 2000, pp. 1503-1509. doi:10.1063/1.480717 